\title{
Voltage Coordination via Communication in Large-Scale Multi-Area Power Systems. Part II: Simulation Results
}

\author{
Mohammad Moradzadeh" a , René Boel ${ }^{2, b}$ \\ ${ }^{1,2}$ Department of Electrical Energy, Systems and Automation, University of \\ Ghent, Technologiepark-Zwijnaarde 914, B-9052 Ghent, Belgium \\ amohammad.moradzadeh@ugent.be, ${ }^{\mathrm{b}}$ rene.boel@ugent.be
}

Keywords: Coordination, optimization, abstraction, voltage control, large-scale multi-area power system

\begin{abstract}
This two-part paper deals with the coordination of the control actions in a network of many interacting components, where each component is controlled by independent control agents. As a case study we consider voltage control in large electric power systems where ever-increasing pressures from the liberalization and globalization of the electricity market has led to partitioning the power system into multiple areas each operated by an independent Transmission System Operator (TSO). Coordination of local control actions taken by those TSOs is a very challenging problem as poorly coordinated operation of TSOs may endanger the power system security by increasing the risk of blackouts. This second part of the paper presents simulation results on a 12-bus 3-area test system, using the distributed model predictive control paradigm in order to design a coordinating model-based feedback controller. Coordination requires that each agent has some information on what the future evolution of its power flows to and from its neighbors will be. It will be shown that how the communication between agents can avoid voltage collapse in circumstances where classical uncoordinated controllers fail.
\end{abstract}

\section{Introduction}

In this second part of the paper we look for a very weak form of coordination, where control agents operate as independently as possible, taking into account however the need for global stability of the system. Large scale networks of interacting systems may become unstable as a result of a local incident, that triggers a sequence of perturbations in the neighboring areas, in case the local controllers of each area would only react to local information. Indeed the control action in one area might cause further perturbations in neighboring areas, and eventually global interactions may lead to collapse of the whole system. In order to avoid such a collapse of the global system, the local control agents must have some way of anticipating how the input port variables generated by neighboring components will evolve in the future. This can only be achieved if the control agents exchange information about their future control actions, and if each local control agent knows enough about the model of its neighbors in order to predict how these future plans will influence the evolution of the variables at the input ports. Using information on the input signals from neighbors that can be expected to occur as a result of the neighbors announced plans, and using the detailed local model of its own component, a local agent can try to optimize the local behavior. This leads in some sense to a dynamic Nash game, where each agent at each decision moment (and the decision moments at different agents may not be the same) assumes that the other players will stick to their announced control plans. Under this assumption one cannot expect that the system will in general perform as well as would be the case if some global supervisor would apply a global feedback control law. However we have shown under limiting conditions that this strategy can stabilize a 
system in cases when a completely decentralized strategy, without any communications, leads to collapse.

This coordination strategy can be applied to many other networks of interacting components where a local perturbation can lead to global performance degradation. Some examples are: traffic lights in an urban traffic network, on-ramp metering in control of freeway traffic taking overflow into neighboring roads into account, flood control where controllable gates can regulate the flow of water.

This paper presents simulation results on a 12-bus 3-area power system as a case study, using the distributed cooperative model-based predictive control paradigm in order to analyze how the communication between agents can avoid voltage collapse in circumstances where classical uncoordinated controllers fail. The time scale of the long-term voltage control of interest for this paper is in the period of several minutes after a disturbance. The long-term dynamics of interest thus are driven typically by Load Tap Changing transformers (LTCs), Over eXcitation Limiter (OXL) of synchronous generators, and exponential recovery loads.

This paper is organized as follows. Section II formulates the Distributed Model Predictive Control (D-MPC) as our coordination methodology, taking into account the abstraction of the neighboring areas. Section III applies the proposed D-MPC coordination scheme to the problem of voltage coordination in 3-area 12-bus power system. The performance of the distributed coordination scheme will be compared with the decentralized uncoordinated scheme, showing its significant performance. Finally, conclusions are provided in section IV.

\section{Coordination Control Design}

\section{A. Available Control Actions}

The voltage control is accomplished, in decreasing order of priority, through switching of Capacitor Banks (CBs) or FACTS devices, adjustment of terminal voltage setpoints of synchronous generators, adjusting parameters of local controllers of LTCs, and load shedding. Short-term voltage control to eliminate rapid voltage variations, at the primary level of hierarchy, is achieved by fast control of reactive power injections from generators and by switching CBs. Long-term voltage control, the secondary level of hierarchy, is mostly achieved through LTC controls, and at an even higher level by load shedding. LTC controls, as the most likely driving mechanism for voltage control but also as a possible cause of voltage collapse in the long-term, is of special interest. LTCs are slowly acting discrete devices. Under traditional deadband control of LTC, the transformer ratio is changed one step at a time if the voltage error at the designated side of the transformer (usually the distribution side) remains outside a deadband longer than a specified time delay [1]. Currently used LTC control strategies are implemented in several ways such as blocking, locking, reversing and setpoint reduction, using local voltage measurements only [2]. However, those heuristic rules may not suffice to face all possible voltage instability scenarios in large-scale power system. According to the literature, uncoordinated operations of LTCs may increase the risk of voltage collapse [3]. Hence, coordination of LTC actions in the large-scale multi-area power systems must be carefully addressed.

\section{B. Control Methodology}

Model Predictive Control (MPC), also called receding/ moving horizon control, uses an estimate of the current state and an explicit model of the plant in order to predict the future output behavior, for a set $\mathrm{u}_{\alpha}, \alpha \in \mathrm{A}$. A is a set of allowable control sequences $\mathrm{u}_{\alpha}(\mathrm{k}), \mathrm{u}_{\alpha}(\mathrm{k}+1), \ldots, \mathrm{u}_{\alpha}(\mathrm{k}+\mathrm{N})$. MPC compares the performance for a finite number of elements $\alpha \in \mathrm{A}$ and selects the best sequence $\alpha^{*}$. The control value $\mathrm{u}_{\alpha^{*}}(\mathrm{k})$ is then applied to the system during the time interval $\left[\mathrm{t}_{\mathrm{k}}, \mathrm{t}_{\mathrm{k}+1}\right)$.

These calculations are repeated, using new observations at the next time step $t_{k+1}$. 
At present, MPC is the most widely used algorithm to deal with multivariable constrained control problems in industry (but not for power systems). Soft or hard operating constraints on both control inputs and controlled outputs can be explicitly represented in the MPC optimization problem. The requirement that a dynamical model must be known is certainly a limitation, especially for electrical power systems. However, the inherent feedback of an MPC provides robustness against modeling errors [4-7].

\section{Distributed MPC (D-MPC) Formulation Taking External Equivalent Models into Account}

Suppose that, for computional and physical reasons but also as a result of the market liberalization, the overall power system has been decomposed into $I$ interacting TSOs. Either one single LTC or a group of several LTCs located in $\mathrm{TSO}_{\mathrm{i}}, \mathrm{i} \in\{1, \ldots, I\}$ is controlled by $\mathrm{MPC}_{\mathrm{i}}$.

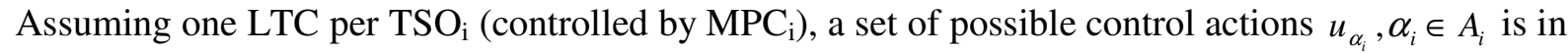
the form of:

$u_{\alpha_{i}}=\{0,+1,-1\}^{N} \times\{0\}^{P+1-N}$ of the form $\left\{u_{\alpha_{i}}(k), u_{\alpha_{i}}(k+1), \ldots, u_{\alpha_{i}}(k+N), 0, \ldots, 0\right\}$.

where 0 refers to having no tap movement, +1 to an upward tap movement, and -1 to a downward tap movement. Note that tap movements are considered only over the control horizon $N$, while no tap movement is considered in the remainder of the prediction in the interval $\left[\mathrm{t}_{\mathrm{k}+\mathrm{N}}, \mathrm{t}_{\mathrm{k}+\mathrm{P}}\right]$. Different combinations of values of $u_{\alpha_{i}}(l), l=k, \ldots, k+N$ define the different possible feedback control input sequences for each LTC in each $\mathrm{TSO}_{\mathrm{i}}$. If $S_{\mathrm{i}}^{\mathrm{k}}$ sequences of tap positions are possible at a given time step $\mathrm{k}$ then $\mathrm{MPC}_{\mathrm{i}}$, knowing only the detailed model of its own area and a reduced model of its neighboring TSOs $j \in I / i$, generates the corresponding $S_{\mathrm{i}}^{\mathrm{k}}$ trajectories and selects the one with lowest local cost that satisfies all local constraints. The controller then applies the first action $u_{\alpha_{i}^{*}}(k)$ of that sequence $u_{\alpha_{i}^{*}}$ letting the system evolve till the next time step $\mathrm{t}_{\mathrm{k}+1}$. furthermore, $\mathrm{TSO}_{\mathrm{i}}$ also sends the selected optimal sequence $u_{\alpha_{i}^{*}}$ of the local control actions (information on the planned tap switching sequences over a finite window in time) to its adjacent TSOs $j \in I / i$. The neighboring TSOs take this information into account in solving their optimization problem for the next time step $t_{k+1}$. This procedure is repeated for each TSO and for each time step.

Assuming that $\mathrm{TSO}_{\mathrm{i}}$ knows at least an approximated model for predicting how control actions of neighbors $j \in I / i$ influence interaction variables (active/reactive power flows through tie-lines, voltages at buses at the end of each tie-lines), this knowledge allows $\mathrm{TSO}_{\mathrm{i}}$ to passively coordinate its action with what neighboring TSOs $j \in I / i$ are planning to do.

Each agent-wise optimization problem can be formulated as a non-linear programming problem of the following form:

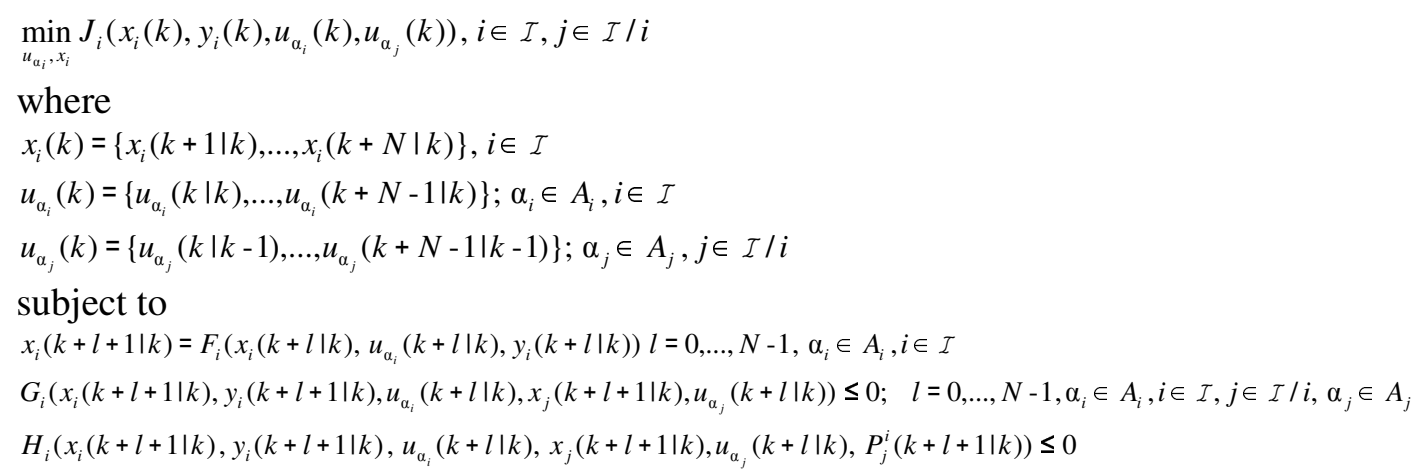


The performance index $J_{i}$ may include a weighted integral of the local bus voltage deviations from their reference values $V_{i}$ in $\mathrm{TSO}_{\mathrm{i}}$, plus a fixed cost per tap changes. $x_{i}, u_{\alpha_{i}}, u_{\alpha_{j}}$ are the sequence of predicted state and control variables in local $\mathrm{TSO}_{\mathrm{i}}$ and its adjacent TSOs $j \in I / i . F_{i}$ is the next-state predictor based on the local system model. $G_{i}$ represents the control specification of maintaining bus voltages within acceptable bounds e.g. $[0.9,1.1]$ p.u. $H_{i}$ is the additionally imposed equality constraint for $\mathrm{TSO}_{\mathrm{i}}$ to model its neighboring TSOs with approximated model parameters $P$. Infinitely fast dynamics, network voltages and currents in load flow equations, are represented by $y$. One can think that exchange of predicted voltage trajectories themselves among TSOs, will require no need to abstract the neighboring areas. We are currently investigating whether this assumption, in the context of competitive electricity market, is feasible.

\section{Simulation Results}

The performance of the proposed distributed coordination methodology has been tested on a 3-area 12-bus power system, for two different scenarios. The system, shown in Fig. 1, is taken from [8]. It consists of three topologically almost identical areas connected together via three double tie lines as transmission system. The generators in Areas 2 and 3 are equipped with AVR and OXL, while area 1 is fed by an $\infty$-bus. The distribution substation in each area is equipped with an LTC and a CB. The agent-wise specification is to keep all bus voltages within the interval e.g. [0.9, 1.1] p.u. by applying the D-MPC methodology to coordinate control actions taken by different LTCs. So we consider the integral of the squared deviation $\left(\mathrm{V}_{\mathrm{t}}-1 \mathrm{p} . \mathrm{u} \text {. }\right)^{2}$ as part of our cost function. It is also highly desirable to minimize the number of LTC moves as tap changes cause transients on the system voltages as well as mechanical wear on the LTC itself. This will be taken care of by adding a term in the cost function penalizing the LTC moves.

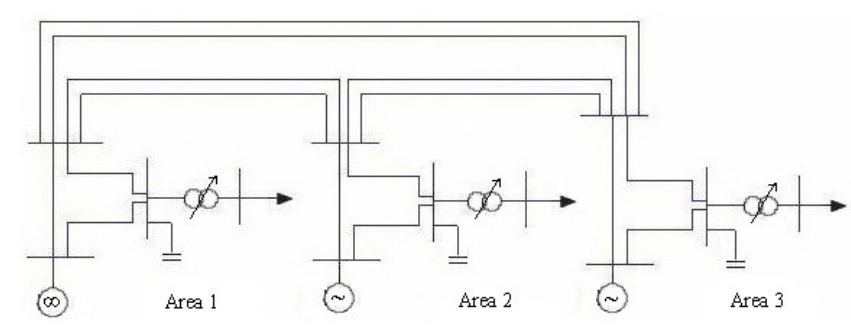

Fig. 1: One-line diagram of a 12-bus power system

\section{A. Line Tripping}

Figures 2 resp. 3 shows the load voltage, LTC and OXL behavior following the tripping of the double tie-line between areas 1 and 3 at $\mathrm{t}=98 \mathrm{~s}$ with decentralized deadband control resp. distributed MPC approach.

\section{A.1. Decentralized Deadband Control}

In this case LTCs operate based on only the local measurements. Tap changes occur, after mechanical time delay, if the voltage at the load bus lies out of deadband for more than 1s. Instability occurs and the solver fails to solve the non-linear equations of the system at $t=470.54 \mathrm{~s}$ when simulation stops. Directly following the fault, load voltages in each area drop, but soon after a short-term equilibrium, with all load voltages settling down close to respective reference voltages, is established. After this point the mechanism driving the system response is OXL and LTC together with load dynamics. After the fault the generator field voltage in area 2 jumps to 2.02 p.u. which exceeds Ifd $\mathrm{lim}_{\mathrm{lim}}=1.88 \mathrm{p}$.u. for this generator. This initiates the inverse time characteristic of the OXL and eventually the OXL is activated at $\mathrm{t}=139 \mathrm{~s}$. At that time the voltage support provided by this 
generator is withdrawn. This results in a further reduction of the load voltage causing the LTC to increase the tap position until its maximum tap limit is reached, and the voltage drops suddenly at $\mathrm{t}=470.54 \mathrm{~s}$ when the generator can no longer deliver the consumed reactive power.

\section{A.2. Distributed MPC Approach}

Now for exactly the same system conditions with all parameters the same as for the previous simulation, but operation of LTCs based on distributed MPC approach, the simulations are repeated. Note that, here the fault detection time of $2 \mathrm{~s}$ is considered and the controller is initiated at $\mathrm{t}=100 \mathrm{~s}$. For the first initialization of the controller, each agent takes the initial tap positions of its neighbor(s) into account. The MPC, with a sampling time of $10 \mathrm{~s}$, control horizon of $30 \mathrm{~s}$ and prediction horizon of $80 \mathrm{~s}$, calculates the optimal control action at $\mathrm{t}=100 \mathrm{~s}$ by simulating the system till $\mathrm{t}=190 \mathrm{~s}$. After solving local optimization problem for each area (one LTC in each area), all LTCs select "no tap movement" as the local optimal control action at $\mathrm{t}=100 \mathrm{~s}$. The procedure is repeated at next sampling instant $\mathrm{t}=110 \mathrm{~s}$ simulating the system till $\mathrm{t}=200 \mathrm{~s}$, again "no tap movement" for all LTCs offers the lowest local cost. The controller is updated every $10 \mathrm{~s}$, till $\mathrm{t}=500 \mathrm{~s}$ and all agents, subject to local cost and local constraints, always prefers having "no tap movement". At $t=500 \mathrm{~s}$, the load voltages are close to their reference values. The OXL for generator in area 3 is not activated at all while the one for generator in area 2 gets activated at $\mathrm{t}=144.4 \mathrm{~s}$, OXL output signal goes up to the value 0.033 p.u. and then stays saturated at 0.029 p.u. Thus one can conclude that the distributed controller avoids the voltage collapse, by predicting the future behavior of the system and communicating among agents.
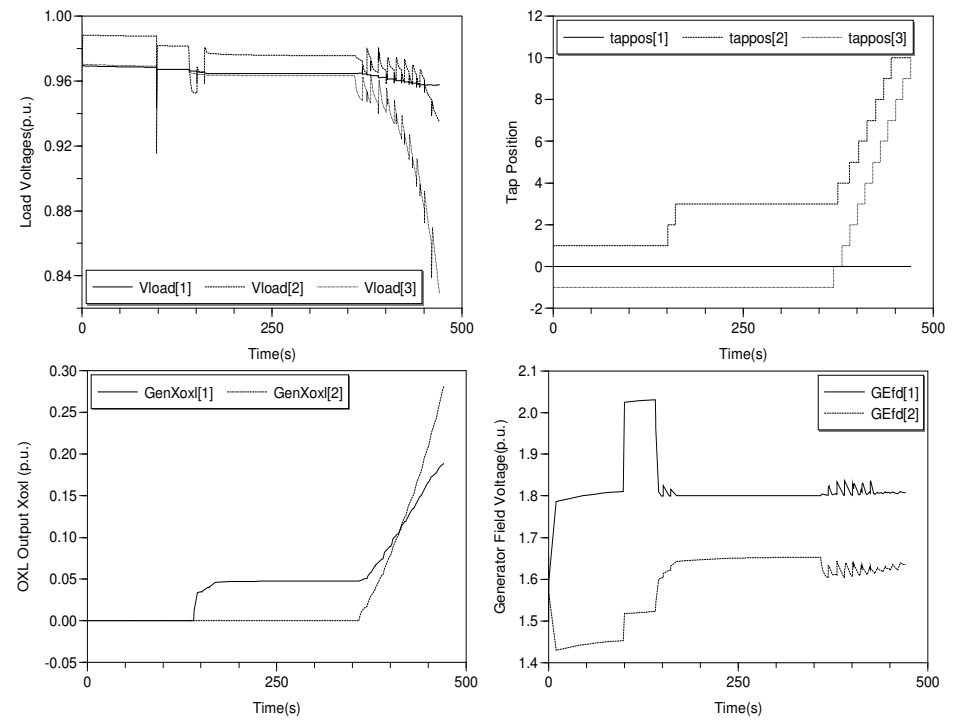

Fig. 2: Response to the line tripping with decentralized control

\section{B. Load Variation}

Load variation is simply considered by suddenly increasing some reactive load in area 2 and decreasing it in area 3 at $t=98 \mathrm{~s}$. Figures 4 resp. 5 shows the system response to this sudden change of reactive load with decentralized deadband control resp. distributed MPC approach.

\section{B.1. Decentralized Deadband Control}

Here the first tap change for LTC in area 1 occurs at $\mathrm{t}=110 \mathrm{~s}$, as it moves one tap down while at the same time the LTC in area 3 moves one tap up. At $\mathrm{t}=154.5 \mathrm{~s}$ the OXL of generator in area 3 gets activated acting as a driving force for 3 subsequent upward tap movements of LTC in area 3 starting at $\mathrm{t}=167 \mathrm{~s}$. The OXL output signal at $\mathrm{t}=400 \mathrm{~s}$ is is $0.0552 \mathrm{p} . \mathrm{u}$. 

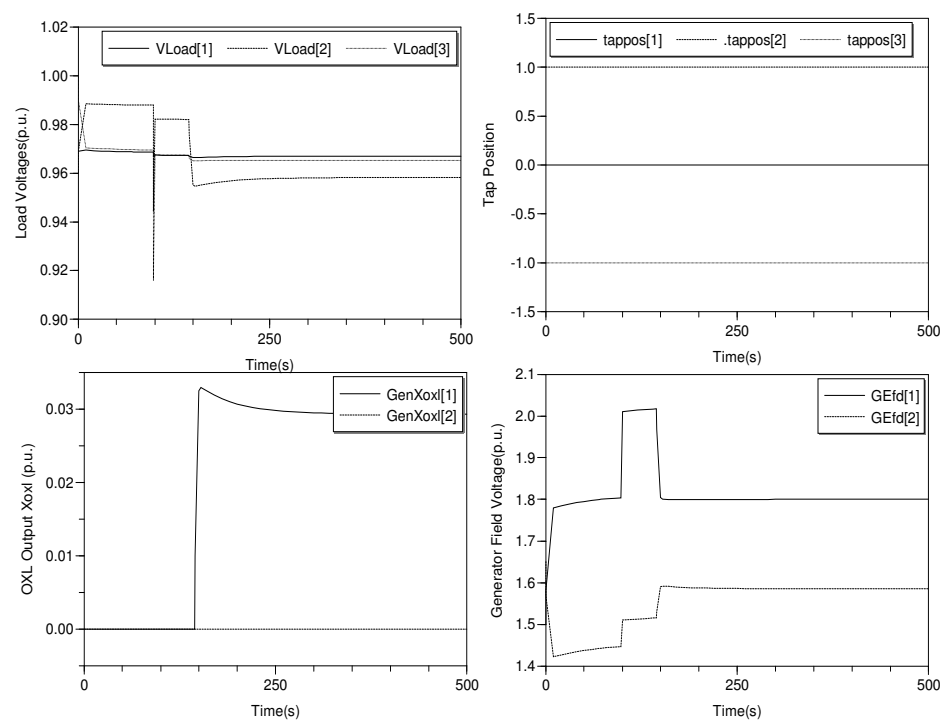

Fig. 3: Response to the line tripping with distributed control
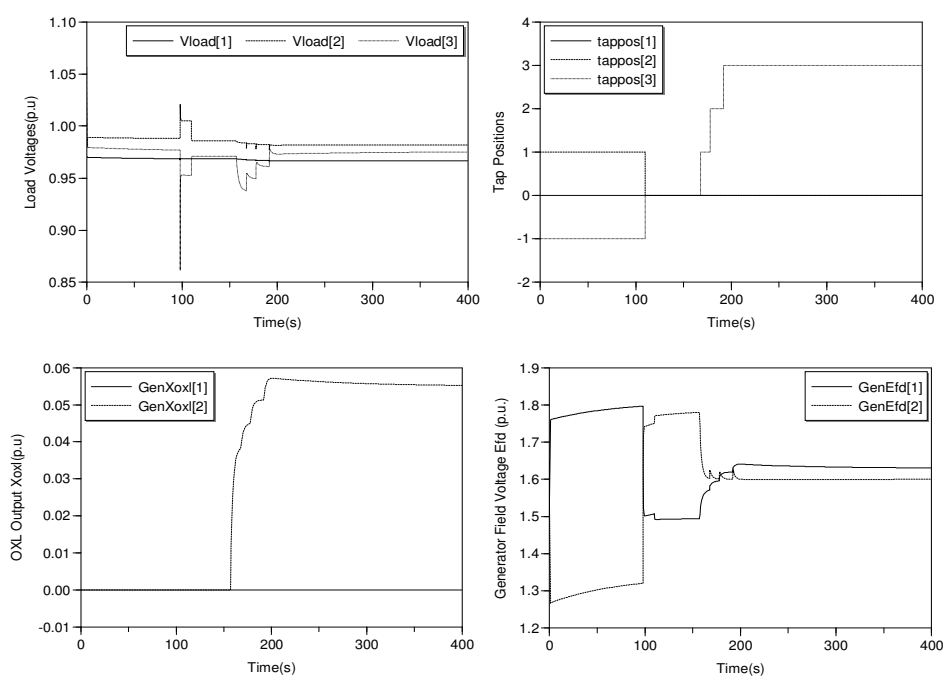

Fig. 4: Response to the load variation with decentralized control

\section{B.2. Distributed MPC Approach}

For exactly the same system conditions, the optimal control actions for LTC in area 3, suggested by the distributed MPC, asks for 4 subsequent upward tap movements at $\mathrm{t}=110,120,130$ and $140 \mathrm{~s}$. At $\mathrm{t}=400 \mathrm{~s}$ all voltages are close to their reference voltage and within the deadband. The OXL output signal at $\mathrm{t}=400 \mathrm{~s}$ is 0.0592 p.u. So it is observed that one less tap movement is required with DMPC as compared to the deadband approach. Note that in the deadband approach, LTC3 initially act on the local voltage at $\mathrm{t}=110 \mathrm{~s}$ and stays in this position till $\mathrm{t}=167 \mathrm{~s}$. At $\mathrm{t}=154.5 \mathrm{~s}$ the OXL2 gets activated and as a result LTC3 again act on the OXL activation while D-MPC does in advance advantageously anticipate the activation of OXL (in this case, $t=150 \mathrm{~s}$ ) and thus start acting early at $\mathrm{t}=110 \mathrm{~s}$. This means that system voltages in D-MPC settles down to their reference values about 50 s earlier than that of deadband approach.

\section{Summary}

This paper, as continuation of the first part, has developed a design methodology for voltage control based on distributed MPC as a tool for coordinating LTCs in adjacent control areas. Via simulation 
on a reasonably sized 12-bus 3-area power system, the paper shows that this coordination control can avoid voltage collapse in cases where traditional uncoordinated decentralized controllers fail. The case study of the distributed MPC considered in this paper deals with a network that is small enough so that each local simulator knows and implements the hybrid dynamical model of the complete system. However for realistic applications the local simulator will only know and implement a detailed mdoel of its own control region, and will need to represent the dynamics of the adjacent regions via more abstract models. This approach is under investigation for the well-known Nordic32 test system. The D-MPC coordination scheme will also be investigated as a tool for designing controllers for medium voltage grids and $\mu$-grids including distributed generation and storage.
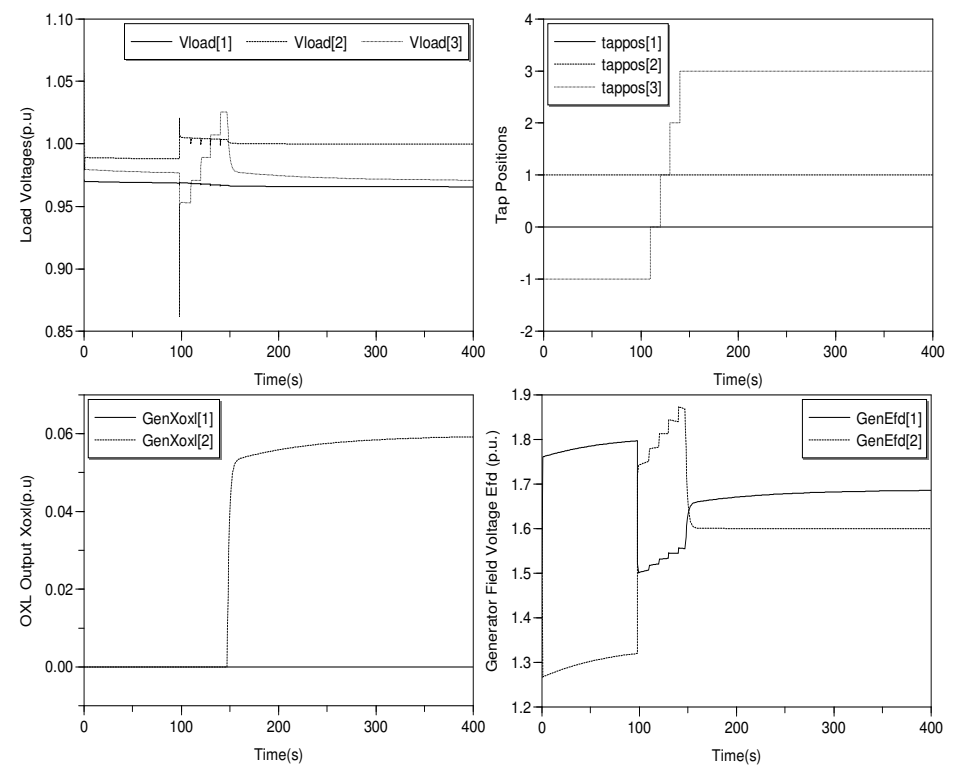

Fig. 5: Response to the load variation with distributed control

\section{References}

[1] T. V. Cutsem and C. Vournas, "Voltage stability of electric power systems", Power electronics and power systems series, Kluwer Academic Publishers, 1998.

[2] F. Capitanescu, B. Otomega, H. Lefebvre, V. Sermanson, T. V. Cutsem "Decentralized tap changer blocking and load shedding against voltage instability: prospective tests on the RTE system", International journal of electrical power and energy systems, 2009.

[3] U.S.-Canada power system outage task force, "Final report on the August 14, 2003 blackout in the United States and Canada:Causes and recommendations", 2004.

[4] J. M. Maciejowski, “Predictive control with constraints”, Pearson Education POD, 2002.

[5] A. N. Venkat, I. A. Hiskens, J. B. Rawlings, S. J. Wright, "Distributed MPC strategies with application to power system automatic generation control", IEEE transactions on control systems technology, vol.16, no.6, pp.1192-1206, Nov. 2008.

[6] R. Scattolini, "Architectures for distributed and hierarchical model predictive control-A review", Journal of process control, 2009.

[7] E. Camponogara, D. Jia, B. H. Krogh, and S. Talukdar, "Distributed model predictive control", IEEE control systems magazine, vol.22, no.1, pp.44-52, Feb.2002.

[8] M. Larsson, "The ABB power transmission test case”, Technical report, 2002. 